\title{
$A b$-Initio Self-Consistent Density Functional Theory Description of Rock-Salt Magnesium Selenide (MgSe)
}

\author{
Blaise Awola Ayirizia1, Yuriy Malozovsky¹, Lashounda Franklin1, Uttam Bhandari², \\ Diola Bagayoko ${ }^{1}$
}

${ }^{1}$ Department of Mathematics and Physics, Southern University and A\&M College, Baton Rouge, LA, USA

${ }^{2}$ Department of Computer Science, Southern University and A\&M College, Baton Rouge, LA, USA

Email: bagayoko@aol.com

How to cite this paper: Ayirizia, B.A., Malozovsky, Y., Franklin, L., Bhandari, U. and Bagayoko, D. (2020) Ab-Initio Self-Consistent Density Functional Theory Description of Rock-Salt Magnesium Selenide (MgSe). Materials Sciences and Applications, 11, 401-414. https://doi.org/10.4236/msa.2020.117027

Received: June 1, 2020

Accepted: June 27, 2020

Published: June 30, 2020

Copyright ( 2020 by author(s) and Scientific Research Publishing Inc. This work is licensed under the Creative Commons Attribution International License (CC BY 4.0).

http://creativecommons.org/licenses/by/4.0/

(c) (i) Open Access

\begin{abstract}
We report results on electronic, transport, and bulk properties of rock-salt magnesium selenide (MgSe), from density functional theory (DFT) calculations. We utilized a local density approximation (LDA) potential and the linear combination of atomic orbitals formalism (LCAO). We followed the Bagayoko, Zhao, and Williams (BZW) method, as enhanced by Ekuma and Franklin (BZW-EF), to perform a generalized minimization of the energy, down to the actual ground state of the material. We describe the successive, self-consistent calculations, with augmented basis sets, that are needed for this generalized minimization. Due to the generalized minimization, our results have the full, physical content of DFT, as per the second DFT theorem [AIP Advances, 4, 127104 (2014)]. Our calculated, indirect bandgap of 2.49 $\mathrm{eV}$, for a room temperature lattice constant of $5.460 \AA$, agrees with experimental findings. We present the ground-state band structure, the related total and partial densities of states, DOS and PDOS, respectively, and electron and hole effective masses for the material. Our calculated bulk modulus of 63.1 $\mathrm{GPa}$ is in excellent agreement with the experimental value of $62.8 \pm 1.6 \mathrm{GPa}$. Our predicted equilibrium lattice constant, at zero temperature, is $5.424 \AA$, with a corresponding indirect bandgap of $2.51 \mathrm{eV}$. We discuss the reasons for the agreements between our findings and available, corresponding, experimental ones, particularly for the band gap, unlike the previous DFT results obtained with ab-initio LDA or GGA potentials.
\end{abstract}

\section{Keywords}

Density Functional Theory, Generalized Energy Minimization, Ground State Band Structure, Band Gap, Related Properties 


\section{Introduction}

Group II-VI semiconductors and their alloys have attracted attention from scientific and technological communities, in recent years, due to their various applications. Specifically, magnesium selenium (MgSe) is an attractive binary semiconductor utilized as a key component in II-VI semiconductor alloys having $\mathrm{n}$ and $\mathrm{p}$ doping abilities [1]. MgSe can exist in four crystalline phases that include rock salt (rs), zinc blende (zb), wurtzite (wz) and nickel arsenide (NiAs) [2] [3]. First principle, self-consistent local density approximation calculations of properties of MgSe show that it is stable in the rock salt phase [3] [4]. Results from several groups show the crystallization of MgSe in cubic (zinc blende) structure from the epitaxial growth on a GaAs substrate by molecular beam epitaxy or Metallo-organic vapor phase epitaxy [2] [5] [6] [7] [8] [9].

Rock salt (rs) MgSe has applications in high-temperature and high-power blue and ultraviolet wavelength optics [2] [3] [10]. It is utilized in photovoltaic devices and in electro-optic crystals with ultra-fast response time spectroscopy. Magnesium selenide (rs-MgSe) has applications in blue-green light-emitting laser diodes (LEDs) [2] [9] [11] [12] and in integrated optical devices [13]. Studies by Falke et al. show that $\mathrm{ZnMgSe}$ alloy has applications in semiconductor optics and in active light-emitting components [14].

Many theoretical studies report an indirect bandgap for MgSe, from $\Gamma$ to $\mathrm{X}$. Drief et al. [15] performed first-principle calculations to determine structural, electronic, elastic and optical properties of MgSe using the Full Potential Linearized Augmented Plane Wave (FP-LAPW) method, in the zinc-blend and rock-salt structures. They found an indirect bandgap $(\Gamma-\mathrm{X})$ of $1.95 \mathrm{eV}$ for the rock-salt structure. Madu and Onwuagba [10] employed the FP-LAPW method, within the Generalized Gradient Approximation (GGA), using the formalism of Perdew, Burke and Ernzerhof [16], to determine electronic and structural properties of rock-salt MgSe. They reported an indirect bandgap of $1.82 \mathrm{eV}$. Kalpana et al. [17] utilized the Tight Binding Linear Muffin-Tin Orbital (TBLMTO) method, with the Local Density Approximation (LDA), to investigate electronic and related properties of MgSe. Their calculations produced an indirect gap of $1.5 \mathrm{eV}$ for rs-MgSe. For rock-salt MgSe, Pandey et al. [18] calculated an indirect bandgap of $4.14 \mathrm{eV}$ by employing the Hartree-Fock method, with correlation corrections. Results from the Full Potential Linear Muffin-tin Orbital (FP-LMTO) computations of Rached et al. [19] showed an indirect bandgap of $1.75 \mathrm{eV}$ for rock salt MgSe. A density functional theory (DFT) calculation by Khan et al. [20], with a modified Becke and Johnson GGA potential and the FP-LAPW method, found an indirect bandgap of $2.83 \mathrm{eV}$ for rs-MgSe. The above calculations and others are summarized in Table 1.

The presumed, experimental band gap for bulk rock-salt $\mathrm{MgSe}$ is $2.47 \mathrm{eV}$, as reported by Heyd et al. [30]. The presumption stems from the fact that these authors compared their calculated band gaps, for bulk rs-MgSe, to this value of $2.47 \mathrm{eV}$, without any mention of films or nanoparticles. We should note that 
Table 1. Previous, calculated, indirect band gaps of rock salt MgSe, mostly using density functional potentials.

\begin{tabular}{|c|c|c|}
\hline Formalism Approach & Potentials & Bandgap Eg $(\mathrm{eV})$ \\
\hline $\begin{array}{l}\text { Full Potential Linearized Augmented Plane Wave } \\
\qquad \text { (FP-LAPW) }\end{array}$ & LDA & $1.95^{\mathrm{a}}$ \\
\hline FP-LAPW & LDA & $1.69^{\mathrm{b}}$ \\
\hline Pseudo Potential approximation & LDA & $1.62^{\mathrm{c}}$ \\
\hline Full-Potential Linear Muffin-Tin Orbitals (FP-LMTO) & LDA & $3.82^{\mathrm{d}}$ \\
\hline Non Local Norm-Conserving Pseudopotentials & LDA & $1.57^{\mathrm{e}}$ \\
\hline FP-LAPW & GGA & $1.70^{\mathrm{f}}$ \\
\hline FP-LAPW & GGA & $1.77^{\mathrm{g}}$ \\
\hline FP-LAPW & GGA & $1.82^{\mathrm{h}}$ \\
\hline Perdew-Burke-Ernzerhof (PBE) & GGA & $1.78^{\mathrm{i}}$ \\
\hline FP-LMTO & GGA & $1.75^{\mathrm{j}}$ \\
\hline Norm-Conserving Pseudo Potentials & GGA-PBE & $1.75^{\mathrm{k}}$ \\
\hline FP-LAPW & $\mathrm{G}_{\mathrm{o}} \mathrm{W}_{\mathrm{o}}$ & $2.68^{1}$ \\
\hline Tight Binding Linear Muffin-Tin Orbital (TB-LMTO) & LDA & $1.5^{\mathrm{m}}$ \\
\hline Pseudopotential Gaussian type & $\mathrm{HF}$ & $10.6^{\mathrm{n}}$ \\
\hline $\begin{array}{l}\text { Linear Combination of Atomic Orbitals-Self } \\
\text { Consistent Field (LCAO-SCF) }\end{array}$ & $\begin{array}{l}\text { HF mixes with } \\
\text { LDA-PZ }\end{array}$ & $8.9^{\circ}$ \\
\hline FP-LAPW & $\mathrm{mBJ}$ LDA & $2.53^{\mathrm{p}}$ \\
\hline FP-LAPW & mBJ GGA & $2.83^{\mathrm{q}}$ \\
\hline Tao-Perdew-Staroverov-Scuseria (TPSS) & mBJ GGA & $2.07^{\mathrm{r}}$ \\
\hline
\end{tabular}

${ }^{a} \operatorname{Ref}$ [15]; ${ }^{\mathrm{k}} \operatorname{Ref}$ [21]; ${ }^{\mathrm{R}} \operatorname{Ref}$ [3]; ${ }^{\mathrm{d}} \operatorname{Ref}$ [19]; ${ }^{\mathrm{e}} \operatorname{Ref}$ [22]; ${ }^{\mathrm{f}} \operatorname{Ref}$ [23]; ${ }^{\mathrm{g}} \operatorname{Ref}$ [24]; ${ }^{\mathrm{h}} \operatorname{Ref}$ [10]; ${ }^{\mathrm{i}} \operatorname{Ref}$ [25]; ${ }^{\mathrm{j}} \operatorname{Ref}$ [19]; ${ }^{\mathrm{k}} \operatorname{Ref}$ [26]; ${ }^{\mathrm{I}} \operatorname{Ref}$ [27]; ${ }^{\mathrm{m}} \operatorname{Ref}$ [17]; ${ }^{\mathrm{N}} \operatorname{Ref}$ [28]; ${ }^{\circ} \operatorname{Ref}$ [29]; ${ }^{\mathrm{P}} \operatorname{Ref}[21] ;{ }^{\mathrm{Q}} \operatorname{Ref}[20] ;{ }^{\mathrm{r}} \operatorname{Ref}[30]$.

some authors [10] [15] [21] [24] who did calculation for rs-MgSe quoted an experimental gap from Fleszar [31] whose calculation was for zb-MgSe. Experimental, optical studies of MgSe thin films, grown with the spray pyrolysis method, found band gap values between 2.45 and $2.75 \mathrm{eV}$ [32]. In Table 2, below, we show these results and others, obtained for films or nanocrystals of rs-MgSe.

$\mathrm{Ab}$-initio LDA potentials led to results that vary from $1.57 \mathrm{eV}$ to $1.95 \mathrm{eV}$, with an outlier of $3.82 \mathrm{eV}$. Ab-initio GGA calculations produced band gaps between 1.75 and $1.82 \mathrm{eV}$. These calculated values, except for the outlier, are much lower than the reported, experimental findings in Table 2. The outlier of $3.82 \mathrm{eV}$ is much larger than the experimental gaps in Table 2. The calculated values obtained with ab-initio DFT potentials are also much smaller than the reported bands for films and nanocrystals. Despite the known enhancement of the band 
Table 2. Experimental band gaps reported for films and nanocrystals of rock salt magnesium selenide (rs-MgSe). The presumed, measured gap for the bulk is as reported by the shown authors.

\begin{tabular}{cc}
\hline Rock Salt Sample Characteristics & Bandgap Eg $(\mathrm{eV})$ \\
\hline $\begin{array}{c}\text { Presumed, experimental band gap of rs-MgSe, } \\
\text { as reported by Heyd et al. [30] }\end{array}$ & 2.47 \\
Nanocrystalline MgSe thin films grown by spray pyrolysis \\
MgSe thin films grown by chemical bath deposition \\
$\begin{array}{c}\text { Nanocrystalline MgSe thin films from spray } \\
\text { solution utilizing spray pyrolysis } \\
\begin{array}{c}\text { Nanocrystalline MgSe thin films deposited } \\
\text { by chemical route triethanolamine } \\
\text { Nanocrystalline MgSe thin films deposited } \\
\text { at room temperature by solution growth }\end{array}\end{array}$ \\
\hline
\end{tabular}

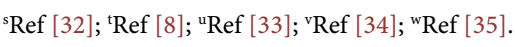

gaps of films and nanocrystals, in comparison to that of the bulk, by quantum confinement, it is not expected to be as large as $0.88 \mathrm{eV}$, the difference between the lowest of the measured gaps $(2.45 \mathrm{eV})$ and the lowest of the calculated ones $(1.57 \mathrm{eV})$. The resolution of this disagreement between experiment and theory is our central motivation for this work. Indeed, the importance of the band gap resides in part in the fact that several properties of materials depend on it, including optical, dielectric, and transport properties. For transport properties, this dependence is partly through the effective masses that are inversely proportional to mobility.

\section{Computational Method and Details for Replication}

In this work, our implementation of the linear combination of atomic orbitals (LCAO) followed the Bagayoko, Zhao, and Williams (BZW) method [36] [37] [38] [39], as enhanced by Ekuma and Franklin (BZW-EF) [40] [41] [42] [43] [44]. We employed the local density approximation (LDA) potential of Ceperley and Alder [45] as parameterized by Vosko et al. [46]. We utilized the General Atomic and Molecular Electronic System Software (GAMESS), a computational package developed at the Ames Laboratory of the United States Department of Energy (DOE), Ames, Iowa [47] [48]. Our self-consistent calculations commence with a small basis set that can account for all the electrons in the system under study. As such, this basis set is not smaller than the minimum basis set. Calculation I is performed with this small basis set. Calculation II is carried out with the basis set of Calculation I, augmented by one atomic orbital. The occupied energies of Calculation I and Calculation II are compared, graphically and numerically, with the Fermi energy set to zero. Some occupied energies from Calculation II are always lower than their corresponding values from Calculation 
I. The second DFT theorem requires the attainment of the ground state through a generalized minimization of the energy. This lowering of the occupied energies shows that the basis set of Calculation I is not sufficient to give the true ground-state description of $\mathrm{MgSe}$. Not having any proof that the eigenvalues from Calculation II are the lowest possible, are compelled to proceed with Calculation III, using the basis set of Calculation II plus one orbital. Then, the occupied energies from Calculations II and III are compared. This process continues until three (3) consecutive calculations (i.e., $\mathrm{N},(\mathrm{N}+1)$, and $(\mathrm{N}+2)$ ) yield the same occupied energies, within our computational uncertainty of $5 \mathrm{meV}$. These three (3) consecutive calculations producing the same occupied energies are the rigorous criterion for the attainment of the absolute minima of the occupied energies, i.e., the ground state of the material. As extensively explained elsewhere [41] [43] [44] the first of these three (3) consecutive calculations is the one providing the DFT description of the material. Its basis set is referred to as the optimal basis set. This basis set is the smallest one that produces the ground state charge density, upon reaching self-consistency. Calculations with an augmented, optimal basis set do not change the occupied energies [36]-[44] [49]; however, the unoccupied energies from these calculations are lower than or equal to their corresponding values produced with the optimal basis set [41] [42] [43] [44]. We address this extra-lowering of some unoccupied energies, while the occupied ones are not changed, in the section on discussion. The quintessential point to underscore here is the fact that values of unoccupied energies that are lowered below their respective values obtained with the optimal basis set no longer belong to the spectrum of the Hamiltonian which is a unique functional of the ground state charge density [41] [42] [43] [44].

The computational details that permit the replication of this work follow. Magnesium selenide, in rock-salt structure, is three-dimensional and crystallizes in the cubic $F m-\overline{3} m$ space group with four (4) formula units of MgSe per unit cell. The $\mathrm{Mg}$ atom is located at the edges and on the six faces. Two inter-penetrating face-centered cubic (FCC) lattices are formed with the Se atom. $\mathrm{Mg}^{2+}$ ions in the crystal structure are bonded to six (6) equivalent $\mathrm{Se}^{2-}$ ions. The positions of the ions of $\mathrm{Mg}$ and Se are $(0,0,0)$ and $(1 / 2,1 / 2,1 / 2)$, respectively.

Our self-consistent calculations were performed utilizing a room temperature, experimental lattice constant of $5.460 \AA$ [50]. We first performed ab-initio, self-consistent calculations for the ionic species, $\mathrm{Mg}^{2+}$ and $\mathrm{Se}^{2-}$. We employed a set of even-tempered Gaussian exponents to expand the radial parts of the atomic wave functions in terms of the Gaussian functions. The number of even-tempered Gaussian exponents used for the s, p, and d orbitals for $\mathrm{Mg}^{2+}$ were 18, 18 and 16, respectively. Likewise, the s, $\mathrm{p}$ and $\mathrm{d}$ orbitals for $\mathrm{Se}^{2-}$. were 18, 18 and 16, respectively. The maximum and minimum exponents used for $\mathrm{Mg}^{2+}$ were $1.1 \times 10^{6}$ and 0.1822 , respectively, whereas the corresponding ones for $\mathrm{Se}^{2-}$ were $0.24 \times 10^{6}$ and 0.135 , respectively. The computational error for the valence charge was $3.289 \times 10^{-4}$ per electron. Self-consistency was reached after 60 
iterations, with $81 \mathrm{k}$-points in the irreducible Broullouin zone, when the difference between the potentials from two consecutive iterations was less than or equal to $10^{-5}$.

\section{Results}

We list below, in Table 3, the valence orbitals in the basis set for the successive, self-consistent calculations, along with the resulting band gaps. Calculation IV is the first of the three consecutive ones (IV, V, and VI) that produce the same, occupied energies. It provides the DFT description of the material; its basis set is the optimal basis set. Our calculated band gap for rock salt MgSe, as produced by Calculation IV, is an indirect one, from $\Gamma-\mathrm{X}$, of $2.49 \mathrm{eV}$.

Figure 1 shows the electronic band structures of rock salt MgSe, as obtained from Calculation IV (solid lines) and Calculation V (dashed lines). As explained in the section on our method, the occupied energies from Calculations IV and V are the same, as shown by the perfect superposition of the valence bands from the two calculations. The unoccupied energies below $4 \mathrm{eV}$ are also the same for the two calculations. For higher energies, the Rayleigh theorem for eigenvalues [36] [37] [38] [39] [41] [42] [43] [44] explains the lowering of some unoccupied energies, in Calculation V, from their corresponding values obtained in Calculation IV.

Table 4 lists the electronic energies at high symmetry points (L-point, $\Gamma$-point, $\mathrm{X}$-point, and K-point) in the Brillouin zone, as obtained with the optimal basis set of Calculation IV. The minimum of the conduction band is at the X-point. The content of this table lends itself to comparison with findings from future experimental studies, including UV and X-ray spectroscopy ones.

Figure 2 and Figure 3, respectively, show the total and partial densities of states energy, DOS and pDOS, respectively, derived from the bands resulting

Table 3. The successive, self-consistent calculations of the BZW-EF method. The basis set is augmented from one calculation to the next. Columns 2 and 3 show the valence orbitals on $\mathrm{Mg}^{2+}$ and $\mathrm{Se}^{2-}$. A superscript of zero (0) indicates an orbital unoccupied in the ionic species. We utilized a room temperature experimental lattice constant of $5.460 \AA$. As explained in the text, Calculation IV provides the DFT description of the material.

\begin{tabular}{ccccc}
\hline $\begin{array}{c}\text { No. of } \\
\text { Calculation }\end{array}$ & $\begin{array}{c}\text { Magnesium }\left(\mathrm{Mg}^{2+}\right) \\
\left(1 \mathrm{~s}^{2} \text {-Core }\right)\end{array}$ & $\begin{array}{c}\text { Selenium }\left(\mathrm{Se}^{2-}\right) \\
\left(1 \mathrm{~s}^{2} 2 \mathrm{~s}^{2} 2 \mathrm{p}^{6}-\mathrm{Core}\right)\end{array}$ & $\begin{array}{c}\text { No. of } \\
\text { functions }\end{array}$ & $\begin{array}{c}\text { Band Gap } \\
(\mathrm{eV})\end{array}$ \\
\hline I & $2 \mathrm{~s}^{2} 2 \mathrm{p}^{6} 3 \mathrm{p}^{0}$ & $3 \mathrm{~s}^{2} 3 \mathrm{p}^{6} 3 \mathrm{~d}^{10} 4 \mathrm{~s}^{2} 4 \mathrm{p}^{6}$ & 40 & $6.20(\Gamma-\mathrm{L})$ \\
II & $2 \mathrm{~s}^{2} 2 \mathrm{p}^{6} 3 \mathrm{p}^{0}$ & $3 \mathrm{~s}^{2} 3 \mathrm{p}^{6} 3 \mathrm{~d}^{10} 4 \mathrm{~s}^{2} 4 \mathrm{p}^{6} 4 \mathrm{~d}^{0}$ & 50 & $6.21(\Gamma-\mathrm{L})$ \\
III & $2 \mathrm{~s}^{2} 2 \mathrm{p}^{6} 3 \mathrm{p}^{0} 3 \mathrm{~s}^{0}$ & $3 \mathrm{~s}^{2} 3 \mathrm{p}^{6} 3 \mathrm{~d}^{10} 4 \mathrm{~s}^{2} 4 \mathrm{p}^{6} 4 \mathrm{~d}^{0}$ & 52 & $2.80(\Gamma-\mathrm{X})$ \\
IV & $2 \mathrm{~s}^{2} 2 \mathrm{p}^{6} 3 \mathrm{p}^{0} 3 \mathrm{~s}^{0}$ & $3 \mathrm{~s}^{2} 3 \mathrm{p}^{6} 3 \mathrm{~d}^{10} 4 \mathrm{~s}^{2} 4 \mathrm{p}^{6} 4 \mathrm{~d}^{0} 5 \mathrm{p}^{0}$ & 58 & $2.49(\Gamma-\mathrm{X})$ \\
V & $2 \mathrm{~s}^{2} 2 \mathrm{p}^{6} 3 \mathrm{p}^{0} 3 \mathrm{~s}^{0} 4 \mathrm{p}^{0}$ & $3 \mathrm{~s}^{2} 3 \mathrm{p}^{6} 3 \mathrm{~d}^{10} 4 \mathrm{~s}^{2} 4 \mathrm{p}^{6} 4 \mathrm{~d}^{0} 5 \mathrm{p}^{0}$ & 64 & $2.50(\Gamma-\mathrm{X})$ \\
VI & $2 \mathrm{~s}^{2} 2 \mathrm{p}^{6} 3 \mathrm{p}^{0} 3 \mathrm{~s}^{0} 4 \mathrm{p}^{0}$ & $3 \mathrm{~s}^{2} 3 \mathrm{p}^{6} 3 \mathrm{~d}^{10} 4 \mathrm{~s}^{2} 4 \mathrm{p}^{6} 4 \mathrm{~d}^{0} 5 \mathrm{p}^{0} 5 \mathrm{~S}^{0}$ & 66 & $2.38(\Gamma-\mathrm{X})$ \\
\hline
\end{tabular}




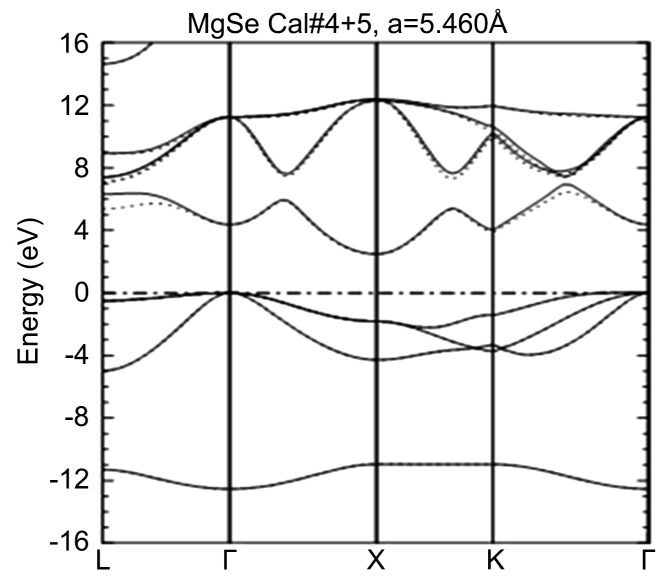

Figure 1. The electronic band structures of rock-salt MgSe, as obtained from Calculation IV (solid lines) and Calculation V (dashed lines), using the BZW-EF method, for a room temperature experimental lattice constant of $5.460 \AA$. The horizontal dashed and dotted line indicates the location of Fermi energy, which is set to zero.

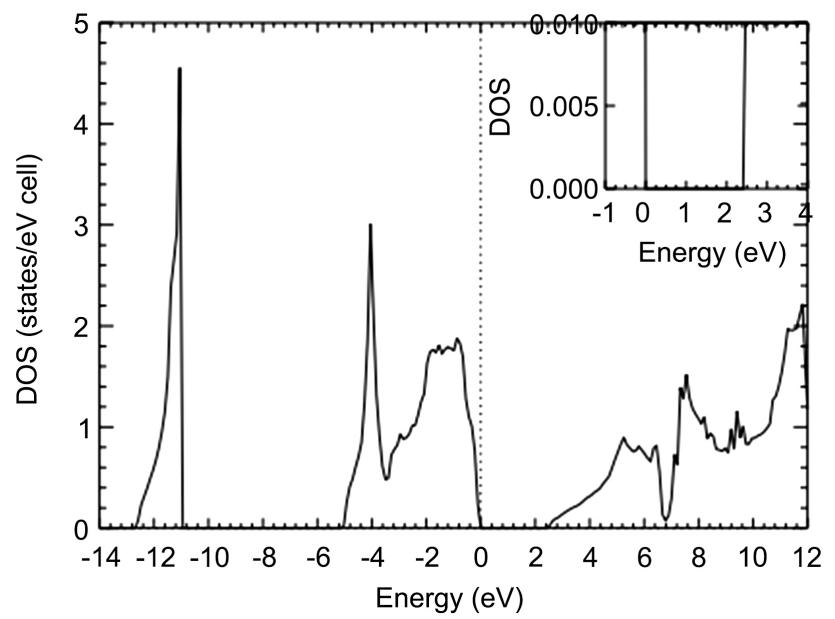

Figure 2. Calculated DOS of rock-salt MgSe obtained from the optimal basis set IV for Calculation IV. The dashed vertical line is the location of Fermi energy $\left(\mathrm{E}_{\mathrm{f}}\right)$ set at zero.

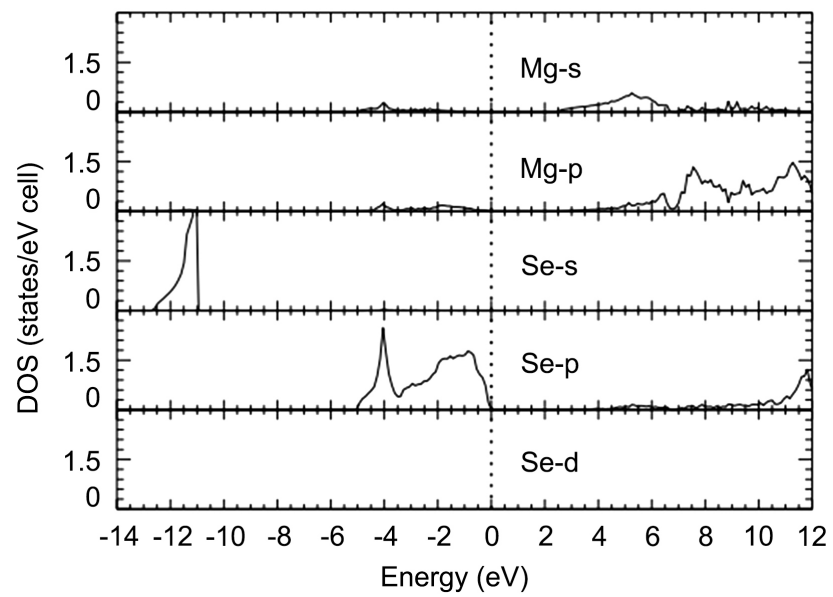

Figure 3. Calculated pDOS of rock salt MgSe obtained from the optimal basis set IV for Calculation IV. The dashed vertical line is the location of Fermi energy $\left(\mathrm{E}_{\mathrm{f}}\right)$ set at zero. 
Table 4. Calculated, electronic energies (in eV) of rock-salt MgSe, at high symmetry points in the Brillouin zone, obtained from Calculation IV. We used the experimental room temperature lattice constant of $5.460 \AA$. The indirect band gap is $\mathrm{E}_{\mathrm{g}}=2.49 \mathrm{eV}$, from $\Gamma$ to $\mathrm{X}$.

\begin{tabular}{cccc}
\hline L-point & Г-point & X-point & K-point \\
\hline 14.654 & 34.882 & 23.504 & 19.386 \\
8.936 & 11.259 & 12.389 & 11.965 \\
7.403 & 11.259 & 12.389 & 10.665 \\
7.349 & 11.259 & 12.293 & 10.267 \\
6.314 & 4.372 & 2.492 & 4.005 \\
-0.503 & 0.000 & -1.816 & -1.431 \\
-0.503 & 0.000 & -1.816 & -3.305 \\
-4.975 & 0.000 & -4.281 & -3.748 \\
-11.302 & -12.535 & -10.956 & -10.954 \\
\hline
\end{tabular}

from Calculation IV, in the energy range of $-14 \mathrm{eV}$ to $12 \mathrm{eV}$. The total valence band width is about $12.61 \mathrm{eV}$. The width of the lowest laying valence band is $1.75 \mathrm{eV}$ while that of the uppermost group of valence bands is $5.0 \mathrm{eV}$. The content of Figure 3 shows that the lowest conduction band is mostly dominated by $\mathrm{Mg}$-s and Mg-p states. The group of uppermost valence bands is mostly from Se-p states, with a feint contribution from Mg-p. The lowest laying valence band is entirely from Se-s, with no other meaningful contributions.

Effective masses are needed for the calculations of several transport properties of materials. These properties include electrical conductivity, mobilities. The latter are inversely proportional to applicable effective masses. For this reason, we calculated electron and hole effective masses for rs-MgSe. We obtained electron effective masses at the conduction band minimum (CBM), at $\mathrm{X}$, and at the lowest point on the conduction band at $\Gamma$. We produced the heavy and light hole effective masses at the valence band maximum (VBM) at $\Gamma$. The calculated effective masses in Table 5 include electron effective mass at the $\Gamma$ point $\left(M_{e \Gamma}\right)$, the longitudinal and transverse electron effective masses at the $\mathrm{X}$ point, $\mathrm{M}_{\mathrm{eXlong}}$ and $M_{\text {extrans, }}$, respectively, and the effective masses for heavy holes 1 and $2\left(M_{h h 1}, M_{h h 2}\right)$ and for the light hole $\left(\mathrm{M}_{\mathrm{lh}}\right)$, in various directions, at the VBM at $\Gamma$. These effective masses are inunits of free-electron mass $\left(\mathrm{m}_{\mathrm{o}}\right)$. At the center of the zone, the electron effective mass at $\Gamma$ is isotropic. While the electron effective masses at $\mathrm{X}$ and the light hole effective masses are mildly anisotropic, the ones for the heavy holes are strongly anisotropic. Along the directions of $(\Gamma-\mathrm{L})_{111}$ and $(\Gamma-\mathrm{X})_{100}$, we obtained the same respective results for the effective masses of heavy hole 1 and heavy hole 2. Due to the splitting of the bands by the Coulomb crystal field, the effective masses of the heavy holes are different in the $(\Gamma-\mathrm{K})_{110}$ direction. We 
could not find any experimental measurements or theoretical calculations for electron and hole effective masses of rock salt MgSe. The results below are predictions expected to be confirmed by experiment.

Figure 4 is a graph of the total energy versus the lattice constant, within a range of $5.30 \AA-5.60 \AA$, with a scale of $0.05 \AA$ units on the horizontal axis. Our predicted value of the equilibrium lattice constant, at zero temperature, is 5.424 $\AA$, with a corresponding zero-temperature, indirect bandgap of $2.51 \mathrm{eV}$. We calculated the bulk modulus to be $63.1 \mathrm{GPa}$. This result is in excellent agreement with the experimentally determined value of $62.8 \pm 1.6 \mathrm{GPa}$ [51].

\section{Discussion}

The large difference between our calculated band gap and the ones previously obtained with ab initio DFT potentials needs an explanation. The detailed description of our method unveiled the crucial difference between BZW-EF calculations, that perform a generalized minimization of the energy, and other DFT calculations. Indeed, most of the other ab initio DFT calculations employ a single basis set to obtain self-consistent results that are assumed to be those for the ground state. Such results, however, are stationary ones among an infinite number of such solutions [37] [41] [43] [44] [52]. In contrast, DFT BZW-EF calculations search for and verifiably reach the ground state of systems under study.

The Rayleigh theorem [41] [52] [53] for eigenvalues ascertain that the results of self-consistent calculations depend on the size of the basis set. As the basis set

Table 5. Calculated effective masses of rock-salt MgSe, in units of the free electron mass $\left(\mathrm{m}_{\mathrm{o}}\right)$.

\begin{tabular}{|c|c|c|c|c|c|c|c|c|c|c|c|}
\hline \multirow{2}{*}{ MeXlong } & \multirow{2}{*}{ MeXtrans } & \multirow{2}{*}{$\mathrm{Me}_{\Gamma}$} & \multicolumn{3}{|c|}{$M_{h h 1}$} & \multicolumn{3}{|c|}{$\mathrm{M}_{\mathrm{hh} 2}$} & \multicolumn{3}{|c|}{$\mathrm{M}_{\mathrm{lh}}$} \\
\hline & & & $(100)$ & (110) & (111) & $(100)$ & (110) & (111) & $(100)$ & (110) & (111) \\
\hline 0.488 & 0.283 & 0.317 & 0.939 & 3.268 & 1.471 & 0.939 & 1.064 & 3.268 & 0.265 & 0.231 & 0.187 \\
\hline
\end{tabular}

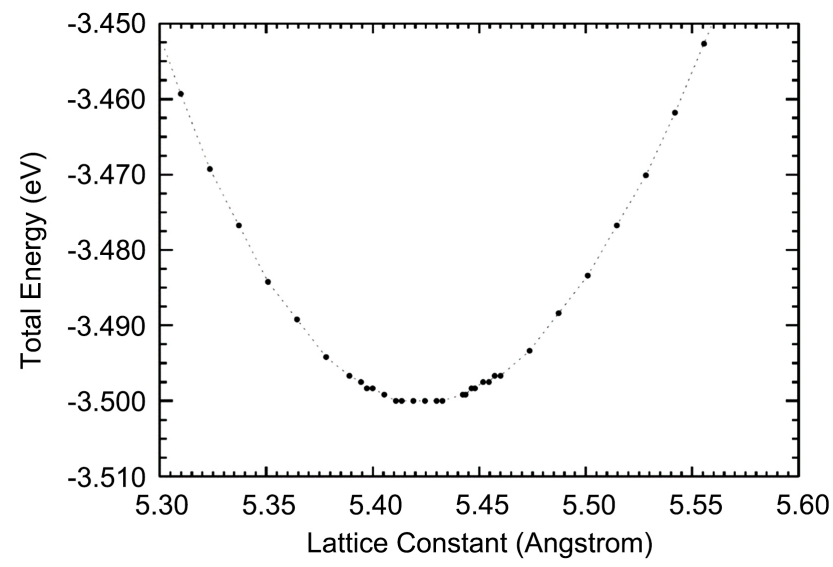

Figure 4. The graph of the total energy versus the lattice constant, for rock-salt MgSe, obtained from our optimal basis set IV of Calculation IV. 
size increases, either a given eigenvalue is lowered or it remains the same. Therefore, once the ground state is reached, further augmentation of the basis set does not affect the occupied energies. However, these larger basis sets lower some unoccupied energies, including the lowest laying ones. This extra-lowering of unoccupied energies, a mathematical artifact [53], while the occupied ones do not change, is a plausible explanation of the widespread underestimation of the band gaps by mainstream DFT calculations. Indeed, single basis set calculations are generally performed with large basis sets, to ensure completeness. Such large single basis sets seem to be over-complete for the description of the ground state, resulting in the spurious lowering of unoccupied energies while the occupied ones do not change.

The referenced attainment of the true ground state of materials guarantees that our results possess the full, physical content of DFT. Consequently, our results agree with available, corresponding experimental ones. Bagayoko [41] [43] discussed our outright predictions of electronic and related properties of $\mathrm{Si}_{3} \mathrm{~N}_{4}$, wurtzite InN, cubic InN, and rutile $\mathrm{TiO}_{2}$; these predictions have been confirmed by experiment [41] [43]. This fact, in addition to our correct description of properties of over 40 semiconductors, portends the likelihood that our calculated effective masses for rs-MgSe will be confirmed by experiment.

\section{Conclusion}

We utilized our well-established BZW-EF computational method to calculate the ground state electronic and related properties of rock salt MgSe. Unlike the previous, ab initio DFT results, our calculated band gap of $2.49 \mathrm{eV}$ agrees with experiment. The same is true for our calculated bulk modulus of $63.1 \mathrm{GPa}$. Our predictions for the effective masses and hybridization patterns, where the latter are derived from the calculated partial densities of states, are expected to be confirmed by experiment.

\section{Acknowledgements}

This work was funded in part by the US Department of Energy, National, Nuclear Security Administration (NNSA) [Award No. DE-NA0002630], the US National Science Foundation (NSF) [Award Nos. EPS-1003897, HRD-1002541], LaSPACE, and LONI-SUBR.

\section{Conflicts of Interest}

The authors declare no conflicts of interest regarding the publication of this paper.

\section{References}

[1] Chad, D.J. (1994) Doping in ZnSe, ZnTe, MgSe, and MgTe Wide-Band-Gap Semieonduetors. Physical Review Letters, 72, 534-538.

https://doi.org/10.1103/PhysRevLett.72.534 
[2] Okuyama, H., Nakano, K., Miyajima, T. and Akimoto, K. (1992) Epitaxial Growth of ZnMgSSe on GaAs Substrate by Molecular Beam Epitaxy. Journal of Crystal Growth, 117, 139-143. https://doi.org/10.1016/0022-0248(92)90732-X

[3] Duman, S., Bağci, S., Tütüncü, H.M. and Srivastava, G.P. (2006) First-Principles Studies of Ground-State and Dynamical Properties of MgS, MgSe, and MgTe in the Rocksalt, Zinc Blende, Wurtzite, and Nickel Arsenide Phases. Physical Review B, 73, 205201. https://doi.org/10.1103/PhysRevB.73.205201

[4] Lee, S.-G. and Chang, K.J. (1995) First-Principles Study of the Structural Properties of MgS-, MgSe-, ZnS-, and ZnSe-Based Superlattices. Physical Review B, 52, 1918-1925. https://doi.org/10.1103/PhysRevB.52.1918

[5] Saib, S., Bouarissa, N., Rodríguez-Hernández, P. and Muñoz, A. (2010) Ab Initio Lattice Dynamics and Piezoelectric Properties of MgS and MgSe Alkaline Earth Chalcogenides. The European Physical Journal B, 73, 185-193.

https://doi.org/10.1140/epjb/e2009-00426-6

[6] Varshney, D., Kaurav, N., Sharma, U. and Singh, R.K. (2008) Phase Transformation and Elastic Behavior of $\mathrm{MgX}(\mathrm{X}=\mathrm{S}, \mathrm{Se}, \mathrm{Te})$ Alkaline Earth Chalcogenides. Journal of Physics and Chemistry of Solids, 69, 60-69. https://doi.org/10.1016/j.jpcs.2007.07.121

[7] Jiang, F., et al. (1998) MOCVD Growth of MgSe Thin Films on GaAs Substrates. Journal of Crystal Growth, 183, 289-293. https://doi.org/10.1016/S0022-0248(97)00426-0

[8] Ubale, A.U. and Sakhare, Y.S. (2013) Physical Properties of MgSe Thin Films Grown by Chemical Bath Deposition Method: Effect of Molar Concentration of $\mathrm{MgCl}_{2}$. Indian Journal of Physics, 87, 1183-1188.

https://doi.org/10.1007/s12648-013-0354-9

[9] Ivanov, S.V., et al. (1998) Molecular Beam Epitaxy of Alternating-Strain ZnSe-Based Multilayer Heterostructures for Blue-Green Lasers. Semiconductors, 32, 1137-1140. https://doi.org/10.1134/1.1187546

[10] Madu, C.A. and Onwuagba, B.N. (2012) Electronic and Structural Properties of MgSe, CaSe, SrSe, and Base. The African Review of Physics, 7, 171-176.

[11] Taniguchi, S., Hino, T., Itoh, S., Nakano, K. and Nakayama, N. (1996) 100h II-VI Blue-Green Laser Diode. Electronics Letters, 32, 552-553. https://doi.org/10.1049/el:19960415

[12] Prete, P., Lovergine, N., Tapfer, L., Zanotti-Fregonara, C. and Mancini, A.M. (2000) MOVPE Growth of MgSe and ZnMgSe on (100)GaAs. Journal of Crystal Growth, 214, 119-124. https://doi.org/10.1016/S0022-0248(00)00045-2

[13] Adetunji, B.I., Adebambom, P.O., Akinlami, J.O. and Adebayo, G.A. (2013) Electroni and Elastic Properties of Zinc-Blende MgSe. International Journal of Modern Physics B, 27, 1350027. https://doi.org/10.1142/S0217979213500276

[14] Falke, U., et al. (1998) Structural Investigations of Polytypes in Zn1-xMgxSe by Transmission Electron Microscopy and Cathodoluminescence. Journal of Crystal Growth, 184-185, 1015-1020. https://doi.org/10.1016/S0022-0248(98)80212-1

[15] Drief, F., Tadjer, A., Mesri, D. and Aourag, H. (2004) First Principles Study of Structural, Electronic, Elastic and Optical Properties of MgS, MgSe and MgTe. Catalysis Today, 89, 343-355. https://doi.org/10.1016/j.cattod.2003.12.013

[16] Perdew, J.P., Burke, K. and Ernzerhof, M. (1996) Generalized Gradient Approximation Made Simple. Physical Review Letters, 77, 3865-3868. https://doi.org/10.1103/PhysRevLett.77.3865 
[17] Kalpana, G., Palanivel, B., Thomas, R.M. and Rajagopalan, M. (1996) Electronic and Structural Properties of MgS and MgSe. Physica B: Condensed Matter, 222, 223-228. https://doi.org/10.1016/0921-4526(96)00014-2

[18] Pandey, R., Lepak, P. and Jaffe, J.E. (1992) Electronic Structure of Alkaline-Earth Selenides. Physical Review B, 46, 4976-4977. https://doi.org/10.1103/PhysRevB.46.4976

[19] Rached, D., Benkhettou, N., Soudini, B., Abbar, B., Sekkal, N. and Driz, M. (2003) Electronic Structure Calculations of Magnesium Chalcogenides MgS and MgSe. Physica Status Solidi Basic Research, 240, 565-573. https://doi.org/10.1002/pssb.200301889

[20] Khan, I., Afaq, A., Rahnamaye Aliabad, H.A. and Ahmad, I. (2012) Transition from Optically Inactive to Active Mg-Chalcogenides: A First Principle Study. Computational Materials Science, 61, 278-282. https://doi.org/10.1016/j.commatsci.2012.04.036

[21] Elsayed, H., Olguín, D., Cantarero, A. and Hernández-Calderón, I. (2015) Ab Initio Structural and Electronic Band-Structure Study of MgSe. Physica Status Solidi Basic Research, 252, 663-669. https://doi.org/10.1002/pssb.201451648

[22] Van Camp, P., Van Doren, V. and Martins, J. (1997) High-Pressure Phases of Magnesium Selenide and Magnesium Telluride. Physical Review B, 55, 775-779. https://doi.org/10.1103/PhysRevB.55.775

[23] Khenata, R., et al. (2012) First-Principles Studies of Ground-State and Dynamical Properties of MgS, MgSe, and MgTe in the Rocksalt, Zinc Blende, Wurtzite, and Nickel Arsenide Phases. Physical Review B, 240, 3865-3868.

[24] Khenata, R., et al. (2017) Phase Stability and Electronic Behavior of MgS, MgSe and MgTe Compounds. Phase Transitions, 90, 929-941. https://doi.org/10.1080/01411594.2017.1302085

[25] Moret, J.-R. (2014) Density Functional Theory Description of II-VI Compounds. Physical Review B, 61, 1-10.

[26] Deng, C.R., Han, X.Y., Wu, H.Y., Yin, P.F. and Chen, Y.H. (2015) Electronic, Elastic and Dynamical Properties of MgSe under Pressure: Rocksalt and Iron Silicide Phase. Philosophical Magazine, 95, 2240-2256. https://doi.org/10.1080/14786435.2015.1047427

[27] Nejatipour, H. and Dadsetani, M. (2015) Excitonic Effects in the Optical Properties of Alkaline Earth Chalcogenides from First-Principles Calculations. Physica Scripta, 90, 85802. https://doi.org/10.1088/0031-8949/90/8/085802

[28] Marinelli, F. and Lichanot, A. (2003) Elastic Constants and Electronic Structure of Alkaline-Earth Chalcogenides. Performances of Various Hamiltonians. Chemical Physics Letters, 367, 430-438. https://doi.org/10.1016/S0009-2614(02)01698-6

[29] Marinelli, F., Dupin, H. and Lichanot, A. (2000) Comparison of Elastic Constants and Electronic Structures in the Series of the Alkaline-Earth Selenides: A Quantum Chemical Approach. Journal of Physics and Chemistry of Solids, 61, 1707-1715. https://doi.org/10.1016/S0022-3697(00)00049-4

[30] Heyd, J., Peralta, J.E., Scuseria, G.E. and Martin, R.L. (2005) Energy Band Gaps and Lattice Parameters Evaluated with the Heyd-Scuseria-Ernzerhof Screened Hybrid Functional. The Journal of Chemical Physics, 123, 174101.

[31] Fleszar, A. (2001) LDA, GW, and Exact-Exchange Kohn-Sham Scheme Calculations of the Electronic Structure of (Formula Presented) Semiconductors. Physical Review B, 64, 245204. https://doi.org/10.1103/PhysRevB.64.245204 
[32] Ubale, A.U. and Sakhare, Y.S. (2014) Growth of Nanocrystalline MgSe Thin Films by Spray Pyrolysis. Vacuum, 99, 124-126. https://doi.org/10.1016/j.vacuum.2013.05.004

[33] Sakhare, Y.S., Thakare, N.R. and Ubale, A.U. (2016) Influence of Quantity of Spray Solution on the Physical Properties of Spray-Deposited Nanocrystalline MgSe Thin Films. St. Petersburg Polytechnical University Journal: Physics and Mathematics, 2, 17-26. https://doi.org/10.1016/j.spjpm.2016.01.005

[34] Ubale, A.U., Sakhare, Y.S., Ibrahim, S.G. and Belkhedkar, M.R. (2013) Structural, Optical and Electric Properties of Nanocrystalline MgSe Thin Films Deposited by Chemical Route Using Triethanolamine as a Complexing Agent. Solid State Sciences, 23, 96-101. https://doi.org/10.1016/j.solidstatesciences.2013.05.016

[35] Ubale, A.U. and Sakhare, Y.S. (2013) Thickness Dependent Physical Properties of Chemically Deposited Nanocrystalline MgSe Thin Films Deposited at Room Temperature by Solution Growth Method. Materials Science in Semiconductor Processing, 16, 1769-1774. https://doi.org/10.1016/j.mssp.2013.06.015

[36] Bagayoko, D., Zhao, G.L., Fan, J.D. and Wang, J.T. (1998) Ab Initio Calculations of the Electronic Structure and Optical Properties of Ferroelectric Tetragonal $\mathrm{BaTiO}_{3}$. Journal of Physics: Condensed Matter, 10, 5645-5655.

https://doi.org/10.1088/0953-8984/10/25/014

[37] Franklin, L., Ekuma, C.E., Zhao, G.L. and Bagayoko, D. (2013) Density Functional Theory Description of Electronic Properties of Wurtzite Zinc Oxide. Journal of Physics and Chemistry of Solids, 74, 729-736. https://doi.org/10.1016/j.jpcs.2013.01.013

[38] Bagayoko, D., Franklin, L. and Zhao, G.L. (2004) Predictions of Electronic, Structural, and Elastic Properties of Cubic InN. Journal of Applied Physics, 96, 4297-4301.

[39] Ekuma, E.C., Franklin, L., Zhao, G.L., Wang, J.T. and Bagayoko, D. (2011) Local Density Approximation Description of Electronic Properties of Wurtzite Cadmium Sulfide (w-CdS). Canadian Journal of Physics, 89, 319-324. https://doi.org/10.1139/P11-023

[40] Bhandari, U., Bamba, C.O., Malozovsky, Y., Franklin, L.S. and Bagayoko, D. (2018) Predictions of Electronic, Transport, and Structural Properties of Magnesium Sulfide (MgS) in the Rocksalt Structure. Journal of Modern Physics, 9, 1773-1784. https://doi.org/10.4236/jmp.2018.99111

[41] Bagayoko, D. (2014) Understanding Density Functional Theory (DFT) and Completing It in Practice. AIP Advances, 4, 127104.

https://doi.org/10.1063/1.4903408

[42] Ekuma, C.E. and Bagayoko, D. (2011) Ab-Initio Electronic and Structural Properties of Rutile Titanium Dioxide. Japanese Journal of Applied Physics, 50, 2-9. https://doi.org/10.1143/JJAP.50.101103

[43] Bagayoko, D. (2014) Comprendre La Théorie De La Fonctionnelle De La Densité Et La Completer Dans La Pratique. Malian Symposium of Applied Sciences (MSAS-214), Researchgate, August 2014, 251-258.

[44] Bagayoko, D. (2016) Understanding the Relativistic Generalization of Density Functional Theory (DFT) and Completing It in Practice. Journal of Modern Physics, 7, 911-919. https://doi.org/10.4236/jmp.2016.79083

[45] Alder, B.J. and Ceperley, D. (1980) Ground State of the Electron Gas by a Stochastic Method. Physical Review Letters, 45, 566-569.

https://doi.org/10.1103/PhysRevLett.45.566 
[46] Vosko, S.H., Wilk, L. and Nusair, M. (1980) Accurate Spin-Dependent Electron Liquid Correlation Energies for Local Spin Density Calculations: A Critical Analysis. Canadian Journal of Physics, 58, 1200-1211. https://doi.org/10.1139/p80-159

[47] Feibelman, P.J., Appelbaum, J.A. and Hamann, D.R. (1979) Electronic Structure of a Ti(0001) Film. Physical Review B, 20, 1433-1443.

https://doi.org/10.1103/PhysRevB.20.1433

[48] D. R. H. B. N. Harmon, W Weber, (1982) Total-Energy Calculations for Si with a First-Principles Linear-Combination-of-Atomic-Orbitals Method. Physical Review B, 25, 1109. https://doi.org/10.1103/PhysRevB.25.1109

[49] Bagayoko, D. and Franklin, L. (2005) Density-Functional Theory Band Gap of Wurtzite InN. Journal of Applied Physics, 97, 123708-123708-5.

[50] De Graef, M. and McHenry, M.E. (2012) Crystal Structure Descriptions. Structure Materials, 1-101.

[51] Ruoff, A.L., et al. (1998) Sevenfold Coordinated MgSe: Experimental Internal Atom Position Determination to $146 \mathrm{GPa}$, Diffraction Studies to $202 \mathrm{GPa}$, and Theoretical Studies to $500 \mathrm{GPa}$. Physical Review Letters, 81, 2723-2726.

https://doi.org/10.1103/PhysRevLett.81.2723

[52] Ekuma, C.E., Jarrell, M., Moreno, J. and Bagayoko, D. (2013) Re-Examining the Electronic Structure of Germanium: A First-Principle Study. Physics Letters A, 377, 2172-2176. https://doi.org/10.1016/j.physleta.2013.05.043

[53] Bagayoko, D. (1983) Contraction of Gaussian Basis Sets and the Total Energy of FCC Copper. International Journal of Quantum Chemistry, 24, 527-535.

https://doi.org/10.1002/qua.560240857 